\title{
The EOS of neutron matter and the effect of $\Lambda$ hyperons to neutron star structure
}

\author{
Stefano Gandolfi* \\ Theoretical Division, Los Alamos National Laboratory, Los Alamos, New Mexico 87545, USA \\ E-mail: stefano@lanl.gov
}

\section{Diego Lonardoni ${ }^{\dagger}$}

National Superconducting Cyclotron Laboratory, Michigan State University, 640 South Shaw Lane, East Lansing, Michigan 48824, USA

Theoretical Division, Los Alamos National Laboratory, Los Alamos, New Mexico 87545, USA

E-mail: lonardonienscl.msu.edu

\begin{abstract}
The structure of neutron stars is determined by the equation of state of the matter inside the star, which relies on the knowledge of nuclear interactions. While radii of neutron stars mostly depend on the equation of state of neutron matter at nuclear densities, their maximum mass can be drastically affected by the appearance of hyperons at higher densities in the inner core of the star. We summarize recent quantum Monte Carlo results on the calculation of the equation of state of neutron matter at nuclear and higher densities. We report about the development of realistic hyperon-nucleon interactions based on the available experimental data for light- and mediumheavy hypernuclei and on the effect of $\Lambda$ hyperons to the neutron star structure.
\end{abstract}

54th International Winter Meeting on Nuclear Physics

25-29 January 2016

Bormio, Italy

\footnotetext{
*Speaker.

$\dagger$ Speaker.
} 


\section{Introduction}

Neutron stars are the most compact and dense objects in the universe, with typical masses $M \sim 1.4 M_{\odot}$ and radii $R \sim 10 \mathrm{~km}$. Their central densities can be several times larger than nuclear saturation density, $\rho_{0}=0.16 \mathrm{fm}^{-3}$, corresponding to the central density of heavy atomic nuclei. Because at such high densities the Fermi energy is in excess of tens of $\mathrm{MeV}$, neutron stars are largely unaffected by thermal effects, and the matter in their interior exhibits the properties of cold matter at extremely high densities, very far from those realized in terrestrial experiments. In the era of multi-messenger astronomical observations neutron stars offer a unique opportunity to test a broad class of theories, from nuclear physics to general relativity.

From the surface to the interior of a neutron star (NS) stellar matter undergoes a number of transitions. From electrons and neutron rich ions in the outer envelopes, the composition is supposed to change to a degenerate gas of neutrons, protons, electrons and muons in the outer core. The composition of neutron stars in the inner core is still to be understood. However, one possible scenario is that at densities larger than few times $\rho_{0}$ new hadronic degrees of freedom, or more exotic phases, are likely to appear.

The knowledge of the equation of state (EOS) of pure neutron matter is an important bridge between the symmetry energy and neutron star properties. The symmetry energy $E_{\text {sym }}$ is the difference of nuclear matter and neutron matter energy and it gives the energy cost of the isospinasymmetry in the homogeneous nucleonic matter. In the last few years the study of $E_{\text {sym }}$ has received considerable attention (see for example Ref. [1] for a recent experimental/theoretical review).

The role of the symmetry energy is essential to understand the mechanism of stability of veryneutron rich nuclei, but it is also related to many phenomena occurring in neutron stars. The stability of matter inside neutron stars is sensitive to $E_{\text {sym }}$ and its first derivative. Neutrons tend to decay to protons through the $\beta$-decay, and the cooling of neutron stars is related to the proton/neutron ratio as a function of the density. This ratio is mainly governed by the behavior of $E_{\mathrm{sym}}$ as a function of the density.

The inner crust of neutron stars, where the density is a fraction of nuclear density, is mostly composed of neutrons surrounding a matter made of extremely-neutron rich nuclei that, depending on the density, may exhibit very different phases and properties. The extremely rich phase diagram of the neutron crustal matter is also related to $E_{\mathrm{sym}}$. For example it governs the phase-transition between the crust and the core [2] and $r$-mode instability [3, 4].

The calculation of the EOS of neutron matter is particularly difficult because neutron matter is one of the most strongly-correlated fermionic systems. Neutron matter is often modeled by density functionals. Traditional Skyrme models (see for example Ref. [5] and references therein) and relativistic mean-field models (see for example Refs. [6,7]) are two general classes of density functional theories. Another class of these calculations uses nuclear potentials, like Argonne and Urbana/Illinois forces, that reproduces two-body scattering and properties of light nuclei with very high precision [8]. For a recent review of neutron matter see Ref. [9].

In this paper we summarize results of the equation of state of pure neutron and $\Lambda$-neutron matter based on quantum Monte Carlo (QMC) methods, that are used to accurately calculate properties of nuclear systems in a non-perturbative framework. 


\section{Nuclear Hamiltonians and quantum Monte Carlo methods}

In our model, nuclei and neutron matter are described by non-relativistic point-like particles interacting via two- and three-body forces:

$$
H_{\mathrm{nuc}}=\sum_{i} \frac{p_{i}^{2}}{2 m_{N}}+\sum_{i<j} v_{i j}+\sum_{i<j<k} v_{i j k}
$$

The two body-potential that we use is the Argonne AV8' [10], that is a simplified form of the Argonne AV18 [11]. Although simpler to use in QMC calculations, the AV8' provides almost the same accuracy as AV18 in fitting NN scattering data. The three-body force is not as well constrained as the $\mathrm{NN}$ interaction, but its inclusion in realistic nuclear Hamiltonians is important to correctly describe the binding energy of light nuclei [8]. The Urbana IX (UIX) three-body force has been originally proposed in combination with the Argonne AV18 and AV8' [12]. Although it slightly underbinds the energy of light nuclei, it has been extensively used to study the equation of state of nuclear and neutron matter [13-15]. In this paper we shall present a study of the neutron matter EOS based on different models of three-neutron forces giving specific values of the symmetry energy $[15,16]$.

For $\Lambda$-hypernuclei and $\Lambda$-neutron matter the Hamiltonian is modified as

$$
H_{\text {hyp }}=H_{\text {nuc }}+\sum_{\lambda} \frac{p_{\lambda}^{2}}{2 m_{\Lambda}}+\sum_{\lambda i} v_{\lambda i}+\sum_{\lambda, i<j} v_{\lambda i j},
$$

where latin indices $i, j$ label nucleons and the greek symbol $\lambda$ is used for $\Lambda$ particles. In the strange sector we adopt explicit $\Lambda \mathrm{N}$ and $\Lambda \mathrm{NN}$ phenomenological interactions analogous to the ArgonneIllinois nucleon-nucleon force [17-20].

In the non-strange sector we employ a simplified interaction in order to make the calculations feasible also for heavier hypernuclei. In particular we use Argonne AV4' two-body interaction [10] plus the central repulsive term of the three-body Urbana IX potential [21]. This choice provides a realistic description of closed shell nuclei up to $A=48$ [22].

The two-body $\Lambda \mathrm{N}$ force is modeled with a Urbana-type potential [23], consistent with the available $\Lambda p$ scattering data

$$
v_{\lambda i}=v_{0}\left(r_{\lambda i}\right)+\frac{1}{4} v_{\sigma} T_{\pi}^{2}\left(r_{\lambda i}\right) \boldsymbol{\sigma}_{\lambda} \cdot \boldsymbol{\sigma}_{i}
$$

while the three-body potential $v_{\lambda i j}$ is written as the sum of $2 \pi$-exchange contributions $v_{\lambda i j}^{2 \pi}=v_{\lambda i j}^{2 \pi, P}+$ $v_{\lambda i j}^{2 \pi, S}$ and a spin-dependent dispersive term $v_{\lambda i j}^{D}$ :

$$
\begin{aligned}
& v_{\lambda i j}^{2 \pi, P}=-\frac{C_{P}}{6}\left\{X_{i \lambda}, X_{\lambda j}\right\} \boldsymbol{\tau}_{i} \cdot \boldsymbol{\tau}_{j}, \\
& v_{\lambda i j}^{2 \pi, S}=C_{S} Z\left(r_{\lambda i}\right) Z\left(r_{\lambda j}\right) \boldsymbol{\sigma}_{i} \cdot \hat{\boldsymbol{r}}_{i \lambda} \boldsymbol{\sigma}_{j} \cdot \hat{\boldsymbol{r}}_{j \lambda} \boldsymbol{\tau}_{i} \cdot \boldsymbol{\tau}_{j}, \\
& v_{\lambda i j}^{D}=W_{D} T_{\pi}^{2}\left(r_{\lambda i}\right) T_{\pi}^{2}\left(r_{\lambda j}\right)\left[1+\frac{1}{6} \boldsymbol{\sigma}_{\lambda} \cdot\left(\boldsymbol{\sigma}_{i}+\boldsymbol{\sigma}_{j}\right)\right] .
\end{aligned}
$$


All the details of the hypernuclear interaction, together with the complete list of parameters, can be found in Refs. [19, 20, 24].

We solve the many-body ground-state using the auxiliary field diffusion Monte Carlo (AFDMC) originally introduced by Schmidt and Fantoni [25]. The main idea of QMC methods is to evolve a many-body wave function in imaginary-time:

$$
\Psi(\tau)=\exp [-H \tau] \Psi_{v}
$$

where $\Psi_{v}$ is a variational ansatz and $H$ is the Hamiltonian of the system. In the limit of $\tau \rightarrow \infty$, $\Psi$ approaches the ground-state of $H$. The evolution in imaginary-time is performed by sampling configurations of the system using Monte Carlo techniques, and expectation values are evaluated over the sampled configurations. For more details see for example Refs. [8, 14, 21].

The Green's Function Monte Carlo (GFMC) method is extremely accurate in the study of properties of light nuclei. The variational wave function includes all the possible spin/isospin states of nucleons and it provides a good variational ansatz to start the projection in the imaginary-time. The exponential growing of this states limits the calculation to ${ }^{12} \mathrm{C}$ [26, 27]. The AFDMC method does not explicitly include all the spin/isospin states in the wave function, but they are instead sampled using the Hubbard-Stratonovich transformation. The calculation can be then extended up to many neutrons, making the simulation of homogeneous matter possible. The AFDMC has proven to be very accurate when compared to GFMC calculation of energies of neutrons confined in an external potential [28].

\section{The equation of state at nuclear densities and the symmetry energy}

The symmetry energy is defined as the energy difference between pure neutron matter and symmetric nuclear matter. The energy of nuclear matter is often expressed as an expansion in even powers of the isospin-asymmetry

$$
E(\rho, x)=E_{0}(\rho)+E_{\mathrm{sym}}^{(2)}(\rho)(1-2 x)^{2}+E_{\mathrm{sym}}^{(4)}(1-2 x)^{4}+\ldots,
$$

where $E$ is the energy per particle, $x=\rho_{p} /\left(\rho_{p}+\rho_{n}\right)$ is the proton fraction, $\rho$ is the density of the system, $E_{\mathrm{sym}}^{(2 n)}$ are coefficients multiplying the isospin asymmetry terms $(1-2 x)^{2 n}$, and $E_{0}(\rho)=$ $E(\rho, x=0.5)$ is the energy of symmetric nuclear matter. The symmetry energy $E_{\mathrm{sym}}$ is given by

$$
E_{\mathrm{sym}}(\rho)=E(\rho, 0)-E_{0}(\rho)
$$

The energy of symmetric nuclear matter at saturation extrapolated from the binding energy of heavy nuclei is $E\left(\rho_{0}\right)=-16 \mathrm{MeV}$. The symmetry energy around saturation $\rho_{0}$ can be expanded as

$$
\left.E_{\mathrm{sym}}(\rho)\right|_{\rho_{0}}=E_{\mathrm{sym}}+\frac{L}{3} \frac{\rho-\rho_{0}}{\rho_{0}}+\ldots
$$

where $L$ is related to the slope of $E_{\mathrm{sym}}$. By combining the above equations, we can easily relate the symmetry energy to the EOS of pure neutron matter at density close to $\rho_{0}$.

We present several EOSs obtained using different models of three-neutron force in Fig. 1. The two solid lines correspond to the EOSs calculated using the NN potential alone and including the 


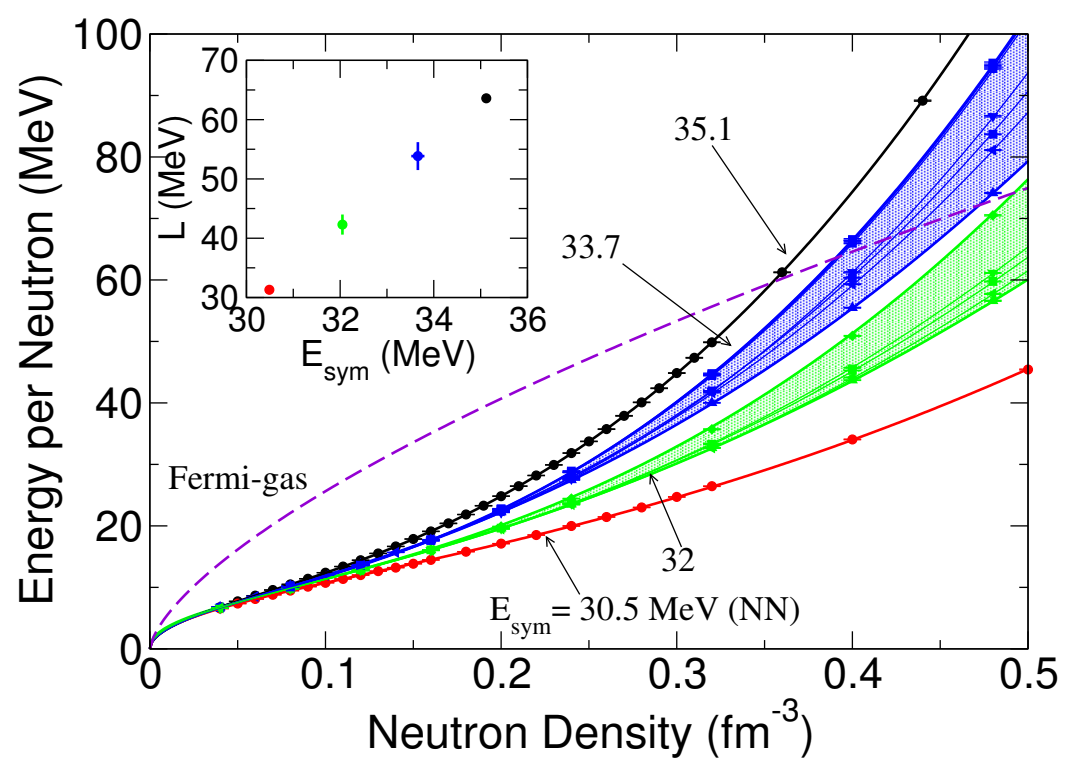

Figure 1: The QMC equation of state of neutron matter for various Hamiltonians. The red (lower) curve is obtained by including the NN (Argonne AV8') alone in the calculation, and the black one is obtained by adding the Urbana IX three-body force. The green and blue bands correspond to EOSs giving the same $E_{\text {sym }}$ (32 and $33.7 \mathrm{MeV}$ respectively), and are obtained by using several models of three-neutron force. In the inset we show the value of $L$ as a function of $E_{\mathrm{sym}}$ obtained by fitting the EOS. The figure is taken from Ref. [15].

UIX three-body force. The effect of using different models of three-neutron force is clear in the two bands, where the high density behavior is shown up to about $3 \rho_{0}$. At such high density, the various models giving the same symmetry energy at saturation produce an uncertainty in the EOS of about $20 \mathrm{MeV}$. The EOS obtained using QMC can be conveniently fit using the following functional [14]:

$$
E(\rho)=a\left(\frac{\rho}{\rho_{0}}\right)^{\alpha}+b\left(\frac{\rho}{\rho_{0}}\right)^{\beta}
$$

where $E$ is the energy per neutron, and $a, b, \alpha$ and $\beta$ are free parameters. The parametrizations of the EOS obtained from different nuclear Hamiltonians is given in Refs. $[15,16]$.

At $\rho_{0}$ symmetric nuclear matter saturates, and we can extract the value of $E_{\mathrm{sym}}$ and $L$ directly from the pure neutron matter EOS. The result of fitting Eq. (3.3) to the pure neutron matter EOS is shown in the inset of Fig. 1. The error bars are obtained by taking the maximum and minimum value of $L$ for a given $E_{\mathrm{sym}}$, and the curves obtained with NN and NN+UIX are thus without error bars. From the plot it is clear that within the models we consider, the correlation between $L$ and $E_{\text {sym }}$ is linear and quite strong. This conclusion is even more evident in Ref. [16] where more different forms of three-body forces have been considered.

\section{Neutron star structure}

When the EOS of the neutron matter has been specified, the structure of an idealized sphericallysymmetric neutron star model can be calculated by integrating the Tolman-Oppenheimer-Volkoff 
(TOV) equations:

$$
\begin{gathered}
\frac{d P}{d r}=-\frac{G\left[m(r)+4 \pi r^{3} P / c^{2}\right]\left[\varepsilon+P / c^{2}\right]}{r\left[r-2 G m(r) / c^{2}\right]}, \\
\frac{d m(r)}{d r}=4 \pi \varepsilon r^{2},
\end{gathered}
$$

where $P=\rho^{2}(\partial E / \partial \rho)$ and $\varepsilon=\rho\left(E+m_{N}\right)$ are the pressure and the energy density, $m_{N}$ is the neutron mass, $m(r)$ is the gravitational mass enclosed within a radius $r$, and $G$ is the gravitational constant. The solution of the TOV equations for a given central density gives the profiles of $\rho, \varepsilon$ and $P$ as functions of radius $r$, and also the total radius $R$ and mass $M=m(R)$. The total radius $R$ is given by the condition $P(R)=0$.

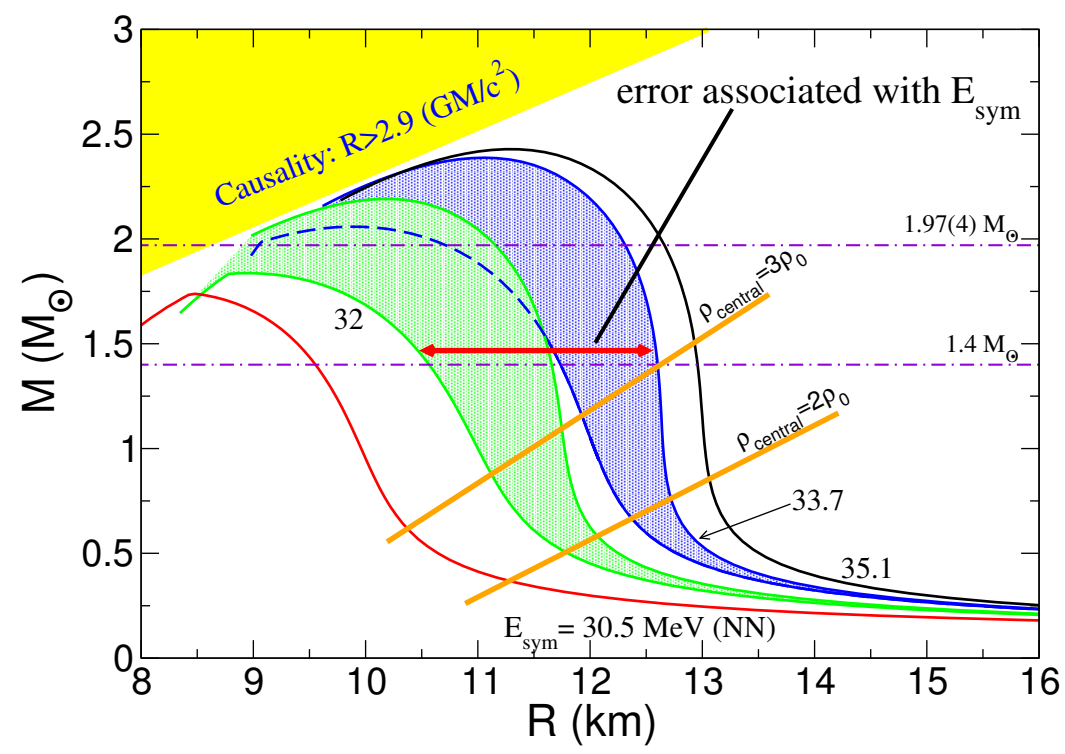

Figure 2: The mass-radius relation of neutron stars obtained from the EOS calculated using QMC. The various colors represent the $M-R$ result obtained from the corresponding EOSs described in Fig. 1. The two horizontal lines show the value of $M=1.4 M_{\odot}$ and 1.97(4) $M_{\odot}$ [29]. The figure is taken from Ref. [15].

The mass of a neutron star as a function of its radius is shown in Fig. 2. The two bands correspond to the result obtained using the two sets of EOS giving the same value of $E_{\mathrm{sym}}$ indicated in the figure. As in the case of the EOS, it is clear that the main source of uncertainty in the radius of a neutron star with $M=1.4 M_{\odot}$ is due to the uncertainty on $E_{\mathrm{sym}}$ rather than the model of the three-neutron force. It has to be noted that we have used the EOS of pure neutron matter without imposing the $\beta$-equilibrium, so in our model we do not have protons. However, the addition of a small proton fraction would only slightly change the radius $R[13,30]$, resulting in a difference smaller than other uncertainties in the EOS that we have discussed.

The EOS of neutron matter and its properties can also be extracted from astrophysical observations [31]. By combining the Bayesian analysis with the model of neutron matter of Eq. (3.4) it is possible to compare the QMC prediction with observations [32] and to extract $E_{\mathrm{sym}}$ and $L$ :

$$
E_{\text {sym }}=a+b+16, \quad L=3(a \alpha+b \beta) .
$$


From neutron stars we obtain the constraints $31.2 \mathrm{MeV}<E_{\text {sym }}<34.3 \mathrm{MeV}$ and $36.6 \mathrm{MeV}<L<$ $55.1 \mathrm{MeV}[32]$ at the 2- $\sigma$ confidence level, in agreement with QMC predictions.

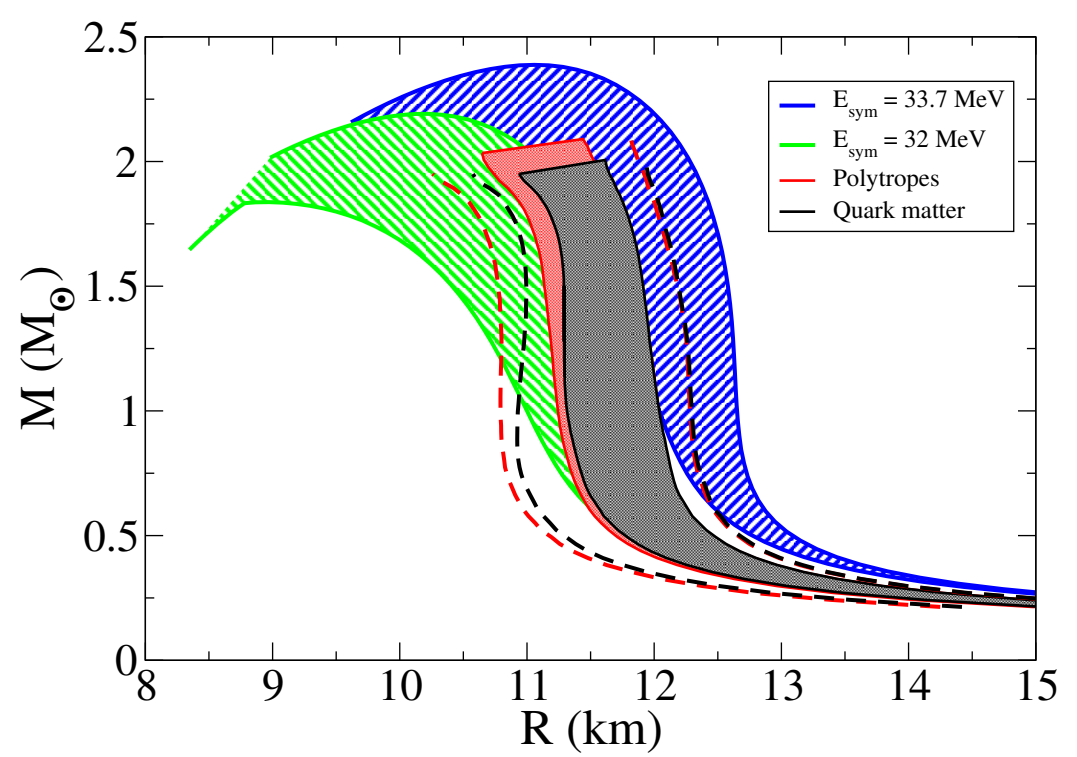

Figure 3: The comparison of the $M-R$ relation of neutron stars obtained from QMC calculations and observations. The blue and green bands are the same as Fig. 2 and correspond to EOSs giving the value of $E_{\mathrm{sym}}$ indicated in the legend. The black and red bands are obtained from neutron star observations of Ref. [32] at the 1- $\sigma$ confidence level (dashed lines at 2- $\sigma$ ), and they correspond to different models of the high-density EOS.

The agreement between theoretical calculations with the neutron star structure obtained from observations is well represented in Fig. 3. The two green and blue bands correspond to the $M-R$ relation obtained from the EOS of Fig. 1, and the black and red bands represent the astrophysical observation of Ref. [32] using different models for the high-density EOS.

\section{Neutron matter at high density}

In Ref. [33] Ambartsumyan and Saakyan reported the first theoretical indication of the appearance of hyperons in the core of a NS. In the degenerate dense matter of the inner core, when the nucleon chemical potential is large enough, the conversion of nucleons into hyperons might become energetically favorable. On the other hand, Pauli blocking would prevent hyperons from decaying by limiting the phase space available to nucleons. This would lead to a reduction of the Fermi pressure exerted by the baryons and to a softening of the EOS, and, as a consequence, the predicted maximum mass of neutron stars would be reduced. However, the recent measurements of the large neutron star mass values of 1.97(4) $M_{\odot}$ [29] and 2.01(4) $M_{\odot}$ [34] require a stiff equation of state. Other NS observations of masses and radii seem to disfavor a very soft EOS of neutron star matter $[31,32,35]$. This seems to contradict the appearance of strange baryons in high-density matter, given what is known at present about the hyperon-nucleon interaction. This apparent inconsistency between NS mass observations and theoretical calculations is a long standing problem known as hyperon puzzle. 
Currently there is no general agreement (even qualitative) among the predicted results for the EOS and the maximum mass of a NS including hyperons. This has to be ascribed to the combination of an incomplete knowledge of the forces governing the system (in the hypernuclear case both two- and three-body ones), and to the concurrent use of approximated theoretical manybody techniques. Some classes of methods extended to the hyperonic sector predict the appearance of hyperons at around $2-3 \rho_{0}$, and a strong softening of EOS, implying a sizable reduction of the maximum mass [36-39]. On the other hand, other approaches suggest much weaker effects arising from the presence of strange baryons in the core of the star [40-45].

The large body of available nucleon-nucleon scattering data allows to derive satisfactory models of two-body nuclear forces, either purely phenomenological or built on the basis of an effective field theory [11, 46, 47]. In the hyperon-nucleon sector, several models of two-body force are available $[48,49]$, and $\Lambda \mathrm{N}$ and $\Sigma \mathrm{N}$ interactions at next-to-leading order in chiral effective field theory have been recently reported [50]. However, they rely on a poor experimental knowledge. Few hyperon-nucleon scattering data are available, and no scattering data exist in the hyperon-hyperon sector. The main reasons of this lack of information lie in the instability of hyperons in the vacuum, and the impossibility of collecting hyperon-neutron and hyperon-hyperon scattering data. This implies that realistic hypernuclear interaction models must also rely on information extracted from the binding energies of hypernuclei.

In Refs. $[22,24,51]$ it has been shown that the repulsive nature of the three-body hyperonnucleon interaction is the key to satisfactorily reproduce the ground state properties of light- to medium-heavy hypernuclei within a unique theoretical framework. By means of a re-fit of the $\Lambda \mathrm{NN}$ force of Eq. (2.4) to the available $\Lambda$ separation energies of closed-shell $\Lambda$-hypernuclei, AFDMC calculations result in a good agreement with experimental data over a wide mass range. In Fig. 4 we show the binding energy of several hypernuclei calculated with AFDMC and compared with a selection of experimental data. The AFDMC results have been obtained by including the two-body $\Lambda \mathrm{N}$ force alone (dashed red upper curve), and together with two models of the $\Lambda \mathrm{NN}$ interaction, $\Lambda N N(I)$ initially proposed by Usmani [17], and $\Lambda$ NN(II) of Ref. [24]. Hypernuclear three-body forces are crucial to qualitatively and quantitatively reproduce the binding energy of hypernuclei. More results, including several excited states of the hyperon, are reported in Fig. 5 [22].

However, parametrizations of the potential predicting relatively small differences in the $\Lambda$ separation energies of hypernuclei give very different results for the properties of the infinite medium [52].

We define the total baryon density $\rho$ and the $\Lambda$ fraction $x$ as:

$$
\rho=\rho_{n}+\rho_{\Lambda}, \quad x=\frac{\rho_{\Lambda}}{\rho},
$$

where $\rho_{n}$ and $\rho_{\Lambda}$ are the neutron and $\Lambda$ densities. The total energy of the $\Lambda$-neutron matter is given by

$$
E_{\Lambda \mathrm{nm}}(\rho, x)=\left[E_{\mathrm{pnm}}((1-x) \rho)+m_{N}\right](1-x)+\left[E_{\mathrm{p} \Lambda \mathrm{m}}(x \rho)+m_{\Lambda}\right] x+f(\rho, x)
$$

where $E_{\mathrm{pnm}}$ is the one defined in Eq. (3.4), $E_{\mathrm{p} \Lambda \mathrm{m}}$ is the non-interacting energy of pure $\Lambda$ matter, and 
the $\Lambda$-neutron part is parametrized as

$$
f(\rho, x)=c_{1} \frac{x(1-x) \rho}{\rho_{0}}+c_{2} \frac{x(1-x)^{2} \rho^{2}}{\rho_{0}^{2}} .
$$

The two coefficients $c_{1}$ and $c_{2}$ have been obtained by fitting AFDMC results of $\Lambda$-neutron matter at various densities $\rho$ and concentrations $x$. At each density, the fraction $x$ as a function of $\rho$ is obtained by imposing chemical equilibrium, and finally, for a given Hamiltonian, an EOS containing neutrons and $\Lambda \mathrm{s}$ in chemical equilibrium is obtained.

The resulting EOSs span the whole regime extending from the appearance of a substantial fraction of hyperons at $\sim 2 \rho_{0}$ to the absence of $\Lambda$ particles in the entire density range of the star. In Fig. 6 we show the AFDMC results obtained for pure neutron matter (same reported in Fig. 1), and with the addition of $\Lambda$ particles interacting with the hypernuclear forces employed for hypernuclei (results reported in Fig. 4). In the inset the neutron and $\Lambda$ fractions are shown. Note that the $\Lambda \mathrm{NN}$ interaction that better reproduces the binding energy of hypernuclei, $\Lambda N N(I I)$ in Fig. 4 and used in Fig. 5, gives a zero $\Lambda$ fraction at least up to $\rho=0.56 \mathrm{fm}^{-3}$, and then the EOS corresponds to the pure neutron matter one up to such a density.

As suggested by the qualitative change of the EOS, the addition of $\Lambda$ hyperons to neutron matter yields to a sizable effect on the predicted neutron star structure (see Fig. 7). The two Hamiltonians that overbind hypernuclei, i.e. $\Lambda \mathrm{N}$ and $\Lambda \mathrm{N}+\Lambda \mathrm{NN}(\mathrm{I})$, produce too low neutron star masses. In particular, in the latter case hyperons appear at around twice saturation density and the predicted maximum mass is less than $1.4 M_{\odot}$. The EOS for the Hamiltonian $\Lambda \mathrm{N}+\Lambda \mathrm{NN}(\mathrm{II})$ is instead

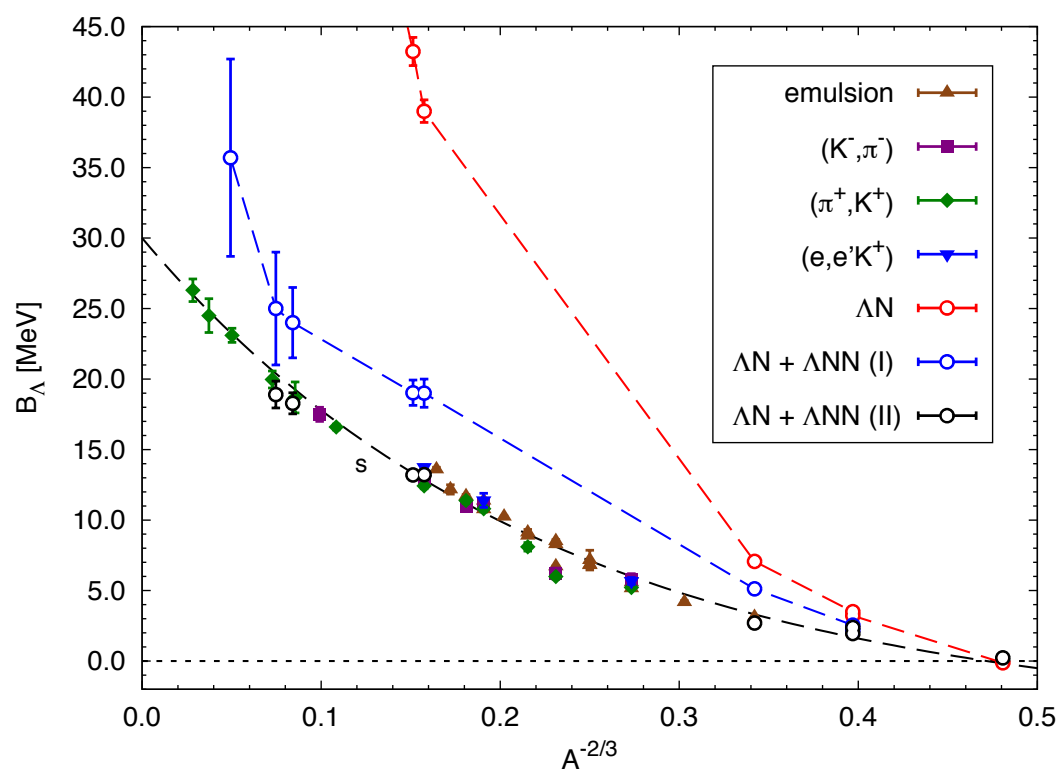

Figure 4: Solid symbols are the available $B_{\Lambda}$ experimental values in $s$ wave for different hypernuclear production mechanisms (see Ref. [22] for the complete list of experimental references). Empty symbols refer to quantum Monte Carlo results. Red dots (upper curve) is the case of two-body $\Lambda \mathrm{N}$ interaction alone. Blue (middle curve) and black (lower curve) dots are the results obtained including two different parametrizations of the three-body hyperon-nucleon force [22, 24]. 


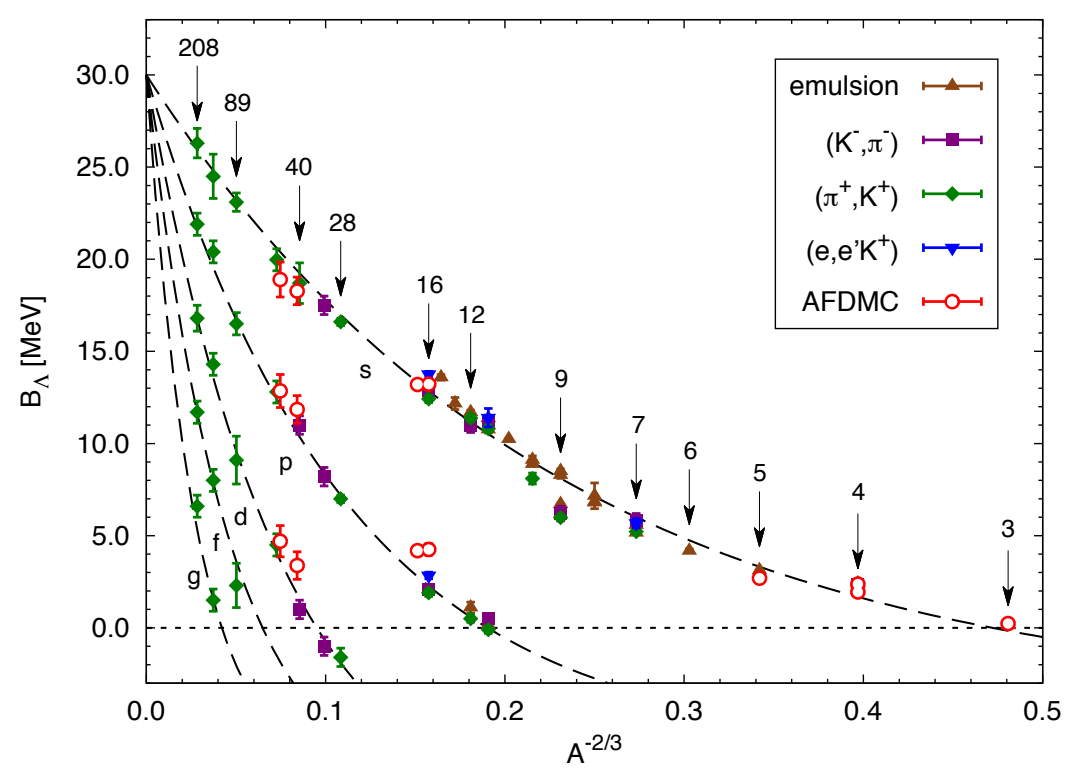

Figure 5: Solid symbols refer to the experimental results for the $\Lambda$ particle in $s, p, d, f$ and $g$ waves. Red empty dots are the quantum Monte Carlo results obtained including the most recent two- plus three-body hyperon-nucleon phenomenological interaction model [22].

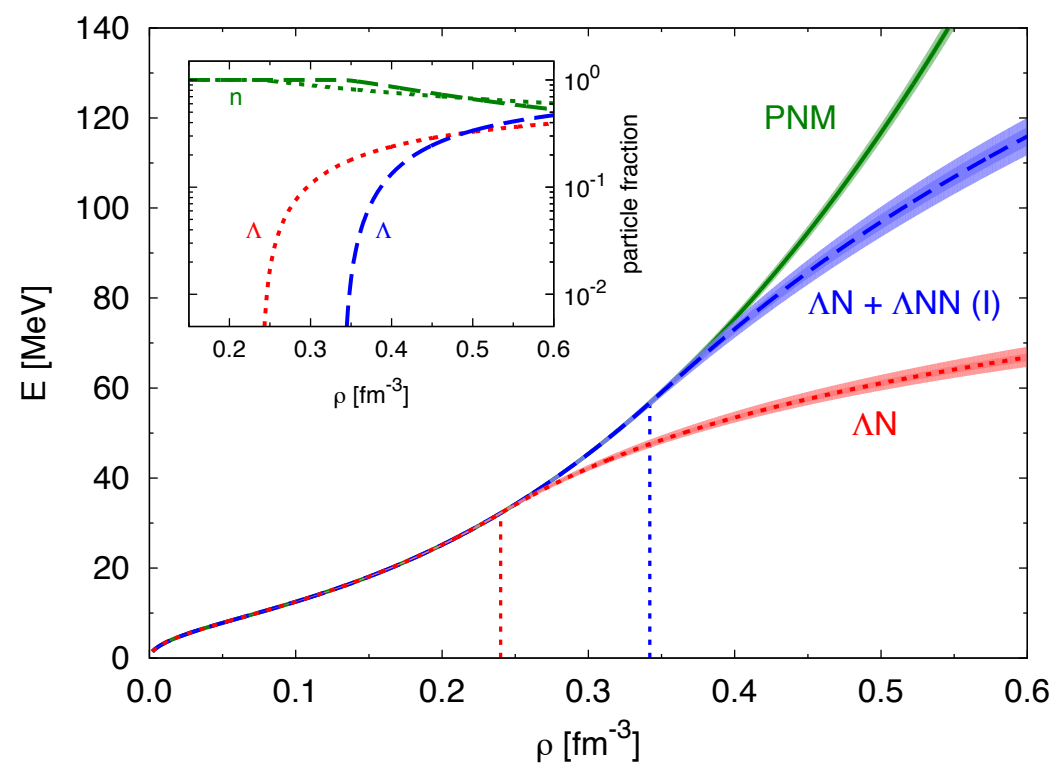

Figure 6: Equations of state. Green solid curves refer to pure neutron matter calculated with realistic twoplus three-nucleon potentials. The red dotted curve represents hyper matter with hyperons interacting via the two-body $\Lambda \mathrm{N}$ force alone. The blue dashed and black dotted-dashed curves are obtained including two different parametrizations of the three-body hyperon-nucleon potential. Shaded regions represent the uncertainties on the results. In the inset, neutron and lambda fractions corresponding to the two hyperneutron matter EOSs. The figure is taken from Ref. [52]. 


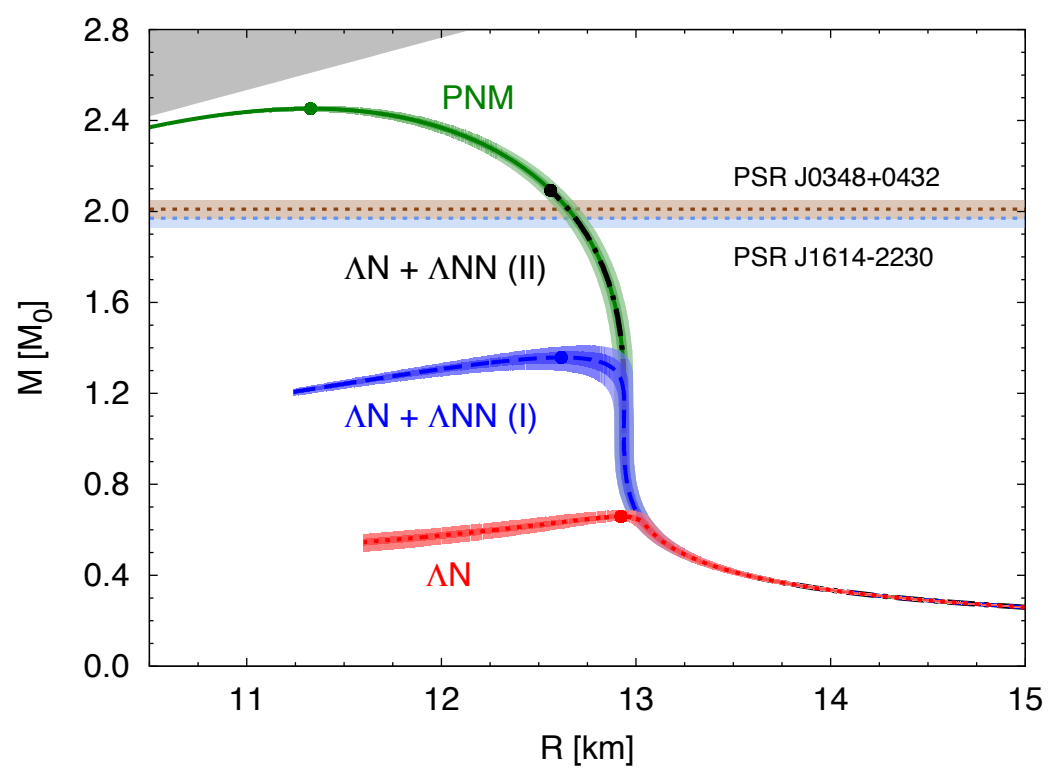

Figure 7: Mass-radius relations. The color scheme is the same as Fig. 6. Full dots represent the predicted maximum masses. Horizontal bands at $\sim 2 M_{\odot}$ are the observed masses of the heavy pulsars PSR J16142230 [29] and PSR J0348+0432 [34]. The grey shaded region is the excluded part of the plot due to causality. The figure is taken from Ref. [52].

stiff enough to support the observations with a lower limit for the predicted maximum mass of $2.09(1) M_{\odot}$.

These results suggest that within the $\Lambda \mathrm{N}$ model that we have considered, the presence of hyperons in the core of the neutron stars cannot be satisfactorily established, and thus there is no clear incompatibility with astrophysical observations when lambdas are included. Therefore, the derivation of realistic hypernuclear potential models is of primary importance to properly assess the role of hyperons to the neutron star structure. This demands a precise and systematic experimental investigation of properties of hypernuclei over a wide range of masses. In this direction a recent study of the isospin dependence of the present three-body hyperon-nucleon force has been carried out, underlying the difficulties in extracting the information on the Hamiltonian from currently available experimental information on hypernuclei [22].

\section{Conclusions}

Quantum Monte Carlo calculations have been extensively used to derive properties of neutron matter at different density regimes to study neutron star structure. The neutron matter EOS around saturation mostly determines the radii of neutron stars [53], and it is directly related to the symmetry energy. We have calculated the EOS, and quantified the connection between $E_{\mathrm{sym}}$ and radii of neutron stars. Although the EOS of neutron matter is qualitatively understood around nuclear density, the appearance of hyperons in the inner core of neutron stars strongly affects the prediction of neutron star properties like the maximum mass. However, by employing hypernuclear interactions that successfully reproduce the experimental separation energies of hypernuclei, the presence of 
hyperons in the core of the neutron stars cannot be satisfactorily established. The hyperon-neutron force will need both additional theoretical investigation and a substantial additional amount of experimental data, in particular for highly asymmetric hypernuclei and excited states of the hyperon.

\section{Acknowledgement}

We would like to thank J. Carlson, F. Catalano, A. Lovato, F. Pederiva, S. C. Pieper, S. Reddy, A. W. Steiner, W. Weise, and R. B. Wiringa for stimulating discussions and for sharing their results. This work was supported by the U.S. Department of Energy, Office of Science, Office of Nuclear Physics, under the NUCLEI SciDAC grant (S.G. and D.L.), by DOE under Contract No. DE-AC5206NA25396 and Los Alamos LDRD grant (S.G.), and under the DOE award DE-SC0013617 titled "FRIB Theory Center - A path for the science at FRIB" (D.L.). This research used resources of the National Energy Research Scientific Computing Center (NERSC), which is supported by the Office of Science of the U.S. Department of Energy under Contract No. DE-AC02-05CH11231, and computing time provided by Institutional Computing (IC) at LANL.

\section{References}

[1] M. B. Tsang, J. R. Stone, F. Camera, P. Danielewicz, S. Gandolfi, K. Hebeler et al., Constraints on the symmetry energy and neutron skins from experiments and theory, Phys. Rev. C 86 (2012) 015803, [nucl-ex/1204.0466].

[2] A. W. Steiner, S. Gandolfi, F. J. Fattoyev and W. G. Newton, Using neutron star observations to determine crust thicknesses, moments of inertia, and tidal deformabilities, Phys. Rev. C 91 (2015) 015804, [nucl-th/1403.7546].

[3] D.-H. Wen, W. G. Newton and B.-A. Li, Sensitivity of the neutron star r-mode instability window to the density dependence of the nuclear symmetry energy, Phys. Rev. C 85 (2012) 025801, [astro-ph.SR/1110.5985].

[4] I. Vidaña, Nuclear symmetry energy and the r-mode instability of neutron stars, Phys. Rev. C 85 (2012) 045808, [nucl-th/1202.4731].

[5] J. R. Stone and P.-G. Reinhard, The Skyrme interaction in finite nuclei and nuclear matter, Prog. Part. Nucl. Phys. 58 (2007) 587, [nucl-th/0607002].

[6] F. J. Fattoyev and J. Piekarewicz, Relativistic models of the neutron-star matter equation of state, Phys. Rev. C 82 (2010) 025805, [nucl-th/1003.1298].

[7] F. J. Fattoyev, W. G. Newton, J. Xu and B.-A. Li, Pure Neutron Matter Constraints and Nuclear Symmetry Energy, Journal of Physics Conference Series 420 (2013) 012108, [nuclth/1209.2718].

[8] J. Carlson, S. Gandolfi, F. Pederiva, S. C. Pieper, R. Schiavilla, K. E. Schmidt et al., Quantum Monte Carlo methods for nuclear physics, Rev. Mod. Phys. 87 (2015) 1067-1118, [nuclth/1412.3081]. 
[9] S. Gandolfi, A. Gezerlis and J. Carlson, Neutron Matter from Low to High Density, Annual Review of Nuclear and Particle Science 65 (2015) 303-328, [nucl-th/1501.05675].

[10] R. Wiringa and S. Pieper, Evolution of Nuclear Spectra with Nuclear Forces, Phys. Rev. Lett. 89 (2002) 18-21, [nucl-th/0207050].

[11] R. B. Wiringa, V. G. J. Stoks and R. Schiavilla, Accurate nucleon-nucleon potential with charge-independence breaking, Phys. Rev. C 51 (1995) 38-51, [nucl-th/9408016].

[12] B. S. Pudliner, V. R. Pandharipande, J. Carlson and R. B. Wiringa, Quantum Monte Carlo Calculations of $A \leq 6$ Nuclei, Phys. Rev. Lett. 74 (1995) 4396-4399, [nucl-th/9502031].

[13] A. Akmal, V. R. Pandharipande and D. G. Ravenhall, Equation of state of nucleon matter and neutron star structure, Phys. Rev. C 58 (1998) 1804, [nucl-th/9804027].

[14] S. Gandolfi, A. Y. Illarionov, K. E. Schmidt, F. Pederiva and S. Fantoni, Quantum Monte Carlo calculation of the equation of state of neutron matter, Phys. Rev. C 79 (2009) 054005, [nucl-th/0903.2610].

[15] S. Gandolfi, J. Carlson and S. Reddy, Maximum mass and radius of neutron stars, and the nuclear symmetry energy, Phys. Rev. C 85 (2012) 032801, [nucl-th/1101.1921].

[16] S. Gandolfi, J. Carlson, S. Reddy, A. W. Steiner and R. B. Wiringa, The equation of state of neutron matter, symmetry energy and neutron star structure, Eur. Phys. J. A 50 (2014) 10, [nucl-th/1307.5815].

[17] A. A. Usmani, S. C. Pieper and Q. N. Usmani, Variational calculations of the $\Lambda$-separation energy of the ${ }_{\Lambda}^{17} \mathrm{O}$ hypernucleus, Phys. Rev. C 51 (1995) 2347, [nucl-th/9502008].

[18] A. A. Usmani, Three-baryon $\lambda$ NN potential, Phys. Rev. C 52 (1995) 1773-1777, [nuclth/9503023].

[19] A. A. Usmani and F. C. Khanna, Behaviour of the $\Lambda \mathrm{N}$ and $\Lambda \mathrm{NN}$ potential strengths in the ${ }_{\Lambda}^{5}$ He hypernucleus, J. Phys. G 35 (2008) 025105, [nucl-th/0612004].

[20] M. Imran, A. A. Usmani, M. Ikram, Z. Hasan and F. C. Khanna, Fully correlated variational Monte Carlo study of ${ }_{\Lambda}^{4} H$ and ${ }_{\Lambda}^{4} H^{*}$ hypernuclei, J. Phys. G 41 (2014) 065101.

[21] B. S. Pudliner, V. R. Pandharipande, J. Carlson, S. C. Pieper and R. B. Wiringa, Quantum Monte Carlo calculations of nuclei with A $\lesssim$, Phys. Rev. C 56 (1997) 1720-1750, [nuclth/9705009].

[22] F. Pederiva, F. Catalano, D. Lonardoni, A. Lovato and S. Gandolfi, New insights on the hyperon puzzle from quantum Monte Carlo calculations, arXiv e-prints 1506.04042 (2015) .

[23] I. Lagaris and V. Pandharipande, Phenomenological two-nucleon interaction operator, Nucl. Phys. A 359 (1981) 331-348. 
[24] D. Lonardoni, F. Pederiva and S. Gandolfi, Accurate determination of the interaction between $\Lambda$ hyperons and nucleons from auxiliary field diffusion Monte Carlo calculations, Phys. Rev. C 89 (2014) 014314, [nucl-th/1312.3844].

[25] K. E. Schmidt and S. Fantoni, A quantum Monte Carlo method for nucleon systems, Phys. Lett. B 446 (1999) 99-103.

[26] S. C. Pieper, Quantum Monte Carlo Calculations of Light Nuclei, Nucl. Phys. A 751 (2005) 516.

[27] A. Lovato, S. Gandolfi, R. Butler, J. Carlson, E. Lusk, S. C. Pieper et al., Charge Form Factor and Sum Rules of Electromagnetic Response Functions in ${ }^{12}$ C, Phys. Rev. Lett. 111 (2013) 092501, [nucl-th/1305.6959].

[28] S. Gandolfi, J. Carlson and S. C. Pieper, Cold Neutrons Trapped in External Fields, Phys. Rev. Lett. 106 (2011) 012501, [nucl-th/1010.4583].

[29] P. B. Demorest, T. Pennucci, S. M. Ransom, M. S. E. Roberts and J. W. T. Hessels, A twosolar-mass neutron star measured using Shapiro delay, Nature 467 (2010) 1081-1083, [astroph.HE/1010.5788].

[30] S. Gandolfi, A. Y. Illarionov, S. Fantoni, J. Miller, F. Pederiva and K. Schmidt, Microscopic calculation of the equation of state of nuclear matter and neutron star structure, Mon. Not. R. Astron. Soc. 404 (2010) L35, [nucl-th/0909.3487].

[31] A. W. Steiner, J. M. Lattimer and E. F. Brown, THE EQUATION OF STATE FROM OBSERVED MASSES AND RADII OF NEUTRON STARS, Astrophys. J. 722 (2010) 33-54, [astro-ph.HE/1005.0811].

[32] A. W. Steiner and S. Gandolfi, Connecting Neutron Star Observations to Three-Body Forces in Neutron Matter and to the Nuclear Symmetry Energy, Phys. Rev. Lett. 108 (2012) 081102, [nucl-th/1110.4142].

[33] V. A. Ambartsumyan and G. S. Saakyan, The Degenerate Superdense Gas of Elementary Particles, Sov. Astron. AJ 4 (oct, 1960) 187.

[34] J. Antoniadis, P. C. C. Freire, N. Wex, T. M. Tauris, R. S. Lynch, M. H. van Kerkwijk et al., A Massive Pulsar in a Compact Relativistic Binary, Science 340 (2013) 1233232, [astroph.HE/1304.6875].

[35] P. F. Bedaque and A. W. Steiner, Hypernuclei and the hyperon problem in neutron stars, Phys. Rev. C 92 (2015) 025803, [nucl-th/1412.8686].

[36] H. DJapo, B.-J. Schaefer and J. Wambach, Appearance of hyperons in neutron stars, Phys. Rev. C 81 (2010) 035803, [nucl-th/0811.2939].

[37] H.-J. Schulze and T. Rijken, Maximum mass of hyperon stars with the Nijmegen ESC08 model, Phys. Rev. C 84 (2011) 035801. 
[38] I. Vidaña, D. Logoteta, C. Providência, A. Polls and I. Bombaci, Estimation of the effect of hyperonic three-body forces on the maximum mass of neutron stars, Europhys. Lett. 94 (2011) 11002, [nucl-th/1006.5660].

[39] E. Massot, J. Margueron and G. Chanfray, On the maximum mass of hyperonic neutron stars, Europhys. Lett. 97 (2012) 39002.

[40] I. Bednarek, P. Haensel, J. L. Zdunik, M. Bejger and R. Mańka, Hyperons in neutron-star cores and a $2 M_{\odot}$ pulsar, Astron. Astrophys. 543 (2012) A157, [astro-ph.SR/1111.6942].

[41] S. Weissenborn, D. Chatterjee and J. Schaffner-Bielich, Hyperons and massive neutron stars: Vector repulsion and SU(3) symmetry, Phys. Rev. C 85 (2012) 065802, [astroph.HE/1112.0234].

[42] W.-Z. Jiang, B.-A. Li and L.-W. Chen, Large-mass Neutron Stars with Hyperonization, Astrophys. J. 756 (2012) 56, [astro-ph.SR/1207.1686].

[43] T. Miyatsu, M.-K. Cheoun and K. Saito, Equation of state for neutron stars in SU(3) flavor symmetry, Phys. Rev. C 88 (2013) 015802, [nucl-th/1304.2121].

[44] N. Gupta and P. Arumugam, Impact of hyperons and antikaons in an extended relativistic mean-field description of neutron stars, Phys. Rev. C 88 (2013) 015803.

[45] Y. Yamamoto, T. Furumoto, N. Yasutake and T. A. Rijken, Hyperon mixing and universal many-body repulsion in neutron stars, Phys. Rev. C 90 (2014) 045805, [nucl-th/1406.4332].

[46] R. Machleidt, F. Sammarruca and Y. Song, Nonlocal nature of the nuclear force and its impact on nuclear structure, Phys. Rev. C 53 (1996) R1483-R1487, [nucl-th/9510023].

[47] A. Gezerlis, I. Tews, E. Epelbaum, S. Gandolfi, K. Hebeler, A. Nogga et al., Quantum Monte Carlo Calculations with Chiral Effective Field Theory Interactions, Phys. Rev. Lett. 111 (2013) 032501, [nucl-th/1303.6243].

[48] T. A. Rijken and Y. Yamamoto, Extended-soft-core baryon-baryon model. II. Hyperonnucleon interaction, Phys. Rev. C 73 (2006) 044008, [nucl-th/0603042].

[49] T. A. Rijken, M. M. Nagels and Y. Yamamoto, Baryon-Baryon Interactions: - Nijmegen Extended-Soft-Core Models -, Progress of Theoretical Physics Supplement 185 (2010) 1471.

[50] J. Haidenbauer, S. Petschauer, N. Kaiser, U.-G. Meißner, A. Nogga and W. Weise, Hyperonnucleon interaction at next-to-leading order in chiral effective field theory, Nucl. Phys. A 915 (2013) 24-58, [nucl-th/1304.5339].

[51] D. Lonardoni, S. Gandolfi and F. Pederiva, Effects of the two-body and three-body hyperonnucleon interactions in $\Lambda$ hypernuclei, Phys. Rev. C 87 (2013) 041303, [nucl-th/1301.7472].

[52] D. Lonardoni, A. Lovato, S. Gandolfi and F. Pederiva, Hyperon Puzzle: Hints from Quantum Monte Carlo Calculations, Phys. Rev. Lett. 114 (2015) 092301, [nucl-th/1407.4448]. 
[53] J. M. Lattimer and M. Prakash, Neutron star structure and the equation of state, Astrophys. J. 550 (2001) 426, [astro-ph/0002232]. 